$$
\text { "kantor" — 2006/6/22 — 18:03 — page } 143 \text { — \#1 }
$$

\title{
Die Methode von Prof. Tibor Szele im Unterricht begabter Schüler
}

\author{
SÁNDOR KÁNTOR
}

Abstract. Prof. Tibor Szele' has attempted to develop the mathematical problemsolving, creativity include the use of investigations and host of other devices beyond the classroom, i.e. in "mathematical circles" for talented students in secondary schools. This paper of the author - who himself has taken part in Seles1s mathematical circles - quotes from these activities according his earlier notes. This description illustrates the didactic method of Prof. T. Szele.

Key words and phrases: didaktische Prinzipien, begabten Förderung, Vektor.

ZDM Subject Classification: B60, C40.

Es ist bekannt, dass die mathematische Ausbildung der begabten Schüler in Ungarn traditionell sehr gut ist. Sehr gute Wettbewerbe, das gute KöMaL (die ungarische mathematische Zeitschrift für Schüler), die guten Lehrer, die vielen Bücher, alles dient der Motivierung. Es ist aber noch die Frage zu beantworten, was ,gut" bedeutet.

Die Pflege der Begabung ist der allgemeinen Didaktik nach dann „gut“, wenn die Themen interessant und die Lösungen geistreich sind.

Unter diesen Voraussetzungen ist eine gute Ausbildung möglich. Dazu, dass die Schüler gute Mathematiker werden, ist eine solche Ausbildung notwendig, die sich speziell mit der Mathematikwissenschaft beschäftigt. Es sind solche Fragen und Denkweisen notwendig, die den werdenden Mathematiker prägen.

Copyright (C) 2006 by University of Debrecen
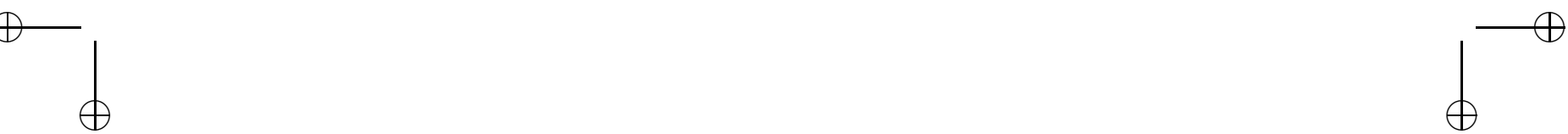


$$
\text { "kantor" — 2006/6/22 — 18:03 — page } 144 \text { — \#2 }
$$

Dazu möchte ich einen Beitrag für Schüler der Mittelschule vorstellen.

Im Jahre 1949 hat Tibor Szele, Mathematikprofessor an der Universität in Debrecen, für Schüler der Mittelschule einen Fachkreis eröffnet. Wöchentlich unterrichtete er zwei Stunden von Januar bis Mai, insgesamt 14 Veranstaltungen.

An diesen Veranstaltungen hatte auch ich teilgenommen. Die dort gemachten Notizen bewahre ich als liebste Erinnerung. Daraus schöpfe ich jetzt. Im Folgenden werde ich einige interessante Probleme aus dem Fachkreis von Szele mit Kommentaren vorstellen. Der Aufbau und die behandelten Probleme dienen auch für heutige Lehrer als Modell.

Neben den Aufzeichnungen gibt es die traurige Tatsache, dass vor jetzt 50 Jahren, im Alter von 37 Jahren Prof. Tibor Szele verstorben ist. Er war schon zu Lebzeiten ein weltberühmter Algebraist und bekam die grösste ungarische Auszeichnung als Wissenschaftler, den Kossuth-Preis. Mit diesem Artikel möchte ich das 50 Järige Jubileum von Sterben Szele Tibor ehren.

Die didaktischen Notizen, bezeichne ich, wenn sie sich auf interessante Themen bzw. geistreiche Lösungen beziechen, mit a-1, a-2, ...; die bezüglichder Mathematik gestellten Fragen bzw. Denkweisen mit b-1, b-2, ...

\section{Die Themen der 14 Fachkreise}

\section{Die Definition und die Eigenschaften der Addition, der Subtraktion und der Multiplikation mit einer Zahl der Vektoren.}

Eine gelöste Aufgabe wurde zu diesem Thema präsentiert.

Man errichtet auf die Seiten eines konvexen Vierecks gleichschenklige, rechtwinklige Dreiecke nach aussen, wobei die Seiten des Vierecks die Hypotenusen der Dreiecke sind. Zu beweisen, dass die rechtwinkligen Ecken der Dreiecke ein solches Viereck bestimmen, dessen Diagonalen senkrecht und gleichlang sind.

Zwei weitere gelöste Aufgaben folgten danach mit anderem Thema:

$\mathrm{Zu}$ beweisen, dass unter Gegenständen zweier verschiedener Farben sowie Formen solche vorkommen, die sich sowohl der Farbe als auch der Form nach unterscheiden.

$\mathrm{Zu}$ beweisen, dass es in einem Graph mit 6 Punkten einen Teilgraph mit 3 Punkten gibt, der ein Vollgraph oder ein Leergraph ist.

Bei den folgenden zwei Aufgaben wurde nur das Ergebnis mitgeteilt:
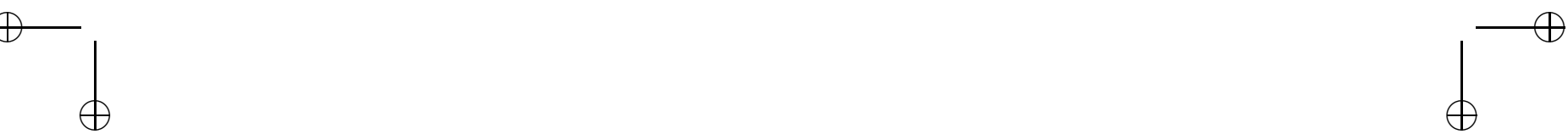


$$
\text { "kantor" — 2006/6/22 — 18:03 — page } 145 \text { — \#3 }
$$

Wieviel Totoscheine müssen ausgefüllt werden, damit bestimmt 12 Zeichen stimmen?

Ermittle die Anzahl der absoluten Permutationen (vgl. Bernoulli - Euler: Die vertauschten Briefe).

\section{Didaktische Informationen:}

a-1) Bei der 1. Beschäftigung dienen die knappe Theorie und die unterschiedlichen Themen der Aufgaben dem allgemeinen Interesse.

a-2) Es ist interessant, dass die leicht verstehbaren, aber auf dieser Ebene schwer zu lösenden Probleme nur für den Abschluss brauchbar und notwendig sind.

b-1) Vektoren wurden in dieser Zeit in der Mittelschule nicht gebraucht, bilden aber eine Grundlage für die höhere Mathematik.

b-2) In der Lösung der ersten Aufgabe wurde die Konstruktion eines neues Begriffs gebraucht: der Drehung eines Vektors. Ein Novum ist in der Mittelschule die Konstruktion eines Begriffs im Laufe der Lösung.

\section{Die Vertiefung und Brauchbarkeit des im Vorhergehenden über Vektoren Gelernten.}

Die folgende Aufgabe kann man mit derselben Methode lösen, die bei der ersten Aufgabe benutzt wurde:

Ein Sechseck $A B C D E F$ ist in einem Kreis mit Radius $r$ eingeschrieben, und es gilt $A B=C D=E F=r$. Zu beweisen, dass die Mittelpunkte der Strecken $B C, D E, F A$ ein gleichseitiges Dreieck bilden.

Thema des Fachkreises war noch die Multiplikation eines Vektors mit einem Skalar; die Berechnung des Vektors, der den Teilungspunkt einer Strecke zeigt, und die Berechnung des Vektors, der den Schwerpunkt eines Dreiecks bzw. Tetraeders zeigt.

\section{Didaktische Informationen:}

a-3) Eine gute Form der Wiederholung ist der Gebrauch der sich ähnelnden Aufgaben.

b-3) Einfache, interessante Anwendungen, die auch in der Theorie wichtig sind. Die neue Theorie wird nach der Lösung einer Aufgabe entwickelt. 


$$
\text { "kantor" — 2006/6/22 — 18:03 — page } 146 \text { — \#4 }
$$

\section{Vektorraum.}

Bei meinen Aufzeichnungen habe ich sehr wenig schriftliches Material. Ich meine, dass bei den Vektorräumen als algebraische Sruktur ein vereinfachter Gebrauch vorgekommen ist und mit vielen Skizzen illustriert wurde. Zum Beispiel ist der folgende Satz interessant: Die Division der Vektoren können wir nicht definieren; parallele Vektoren sind dividierbar.

Didaktische Informationen:

b-4) Zur Entstehung der abstrakten Form werden konkrete Objekte vorbereitet.

4. Funktionen: Polynom, Quadratwurzel, eine Funktion, die in einer komplizierten Form gegeben ist; die Gleichung des Kreises.

Die Methode: die Herstellung einiger Punkte der Graphen der Funktion; die Erkenntnis der Eigenschaften der Funktion nach der Anschauung.

\section{Didaktische Informationen:}

a-4) Auf Grund der Beispiele verstehen die guten Schüler das Wesen des Themas.

5. Die Fortsetzung des Themas der Funktionen: gebrochenrationale Funktion, implizite Funktion $(F(x, y)=0)$, Exponentialfunktion. Die Zahl $e$.

Es waren nur Illustrationen für die Typen der Funktionen.

Die Einführung der Zahl $e$ wurde durch die Berechnung der Summe, auf welche $1 \mathrm{Ft}$ in $1 \mathrm{Jahr}$, mit Zinseszinsen, bei stetiger Kapitalisierung angewachsen ist, eingeführt $\left(\lim _{n \rightarrow \infty}\left(1+\frac{1}{n}\right)^{n}\right)$.

Die Folge $a_{n}=\left(1+\frac{1}{n}\right)^{n}$ ist streng monoton wachsend, nach oben beschränkt. Die Folge $b_{n}=\left(1+\frac{1}{n}\right)^{n+1}$ ist streng monoton fallend, nach unten beschränkt.

\section{Didaktische Informationen:}

b-5) Die Abstraktion eines alltäglichen Begriffs ist sehr lehrhaft.

b-6) Die neuen und abstrakten Begriffe sind auf Grund der Beispiele einfach.

b-7) Statt Beweise sind die Beispiele hinrechend. 


$$
\text { "kantor" — 2006/6/22 — 18:03 — page } 147 \text { — \#5 }
$$

6. Die Zahl $e$ und die Funktion $e^{x}$.

$a_{n} \leq b_{n}, b_{n}-a_{n}=\frac{1}{n}\left(1+\frac{1}{n}\right)<\frac{2}{n} . \lim _{n \rightarrow \infty}\left(1+\frac{1}{n}\right)^{n}$ ist eine Zahl, die wir mit dem Buchstaben $e$ bezeichnen. $\lim _{n \rightarrow \infty}\left(1+\frac{x}{n}\right)^{n}=e^{x}$.

Didaktische Informationen:

b-8) Die den Grenzwert erreichenden Formen der Anschauung und das sich auf die Anschauung gründende Verhalten werden gebraucht. Das kann dann der Schüler verstehen und gebrauchen.

\section{Auseinandersetzung mit zwei Thesen.}

Für die Mittelschule werden sie nicht gebraucht, aber für die höhere Mathematik sind es sehr interessante Thesen:

Die Zahl 1 entsteht als die Summe von verschiedenen (mindenstens drei) Stammbrüchen.

Von allen einem gegebenen spitzwinkligen Dreieck einbeschriebenen Dreiecken hat das Dreieck der Höhenfusspunkte den kleinsten Umfang.

Didaktische Informationen:

a-5) Bei den einzelnen Beschäftigungen wechseln oft die Themen.

b-9) Bei der Vorbereitung der wichtigsten Verhandlungen ist die Auswahl des neuen Themas zu beachten.

\section{Aufgabe zur Berechnung des Extremwertes der Funktionen.}

Der allgemeine Begriff, die Art und die Berechnung des Extremwertes. Eine typische Aufgabe:

Aus einem quadratischen Karton mit den Seitenlängen $a$ ist durch Ausschneiden von Quadraten der Seitenlänge $x$ an den Ecken und anschliessendes Aufbiegen der Seitenwände eine quadratförmige, oben offene Schachtel herzustellen. Wie gross muss $x$ gewählt werden, damit das Volumen der Schachtel maximal wird?

Didaktische Informationen:

b-10) Die Berechnung ist wichtig und vielseitig (auch mit der Differentialrechnung).

\section{Zwei Aufgaben zu geometrischen Extremwerten.}

Für einen gegebenen Kreis das einbeschriebene Dreieck von grösstem Umfang zu bestimmen. 


$$
\text { "kantor" — 2006/6/22 — 18:03 — page } 148 \text { — \#6 }
$$

Den Punkt zu finden, bei dem die Summe seiner Abstände von den Ecken eines gegebenen Dreiecks möglichts klein wird.

Diese berühmte Aufgabe wurde von dem französischen Mathematiker Fermat (1601-1665) dem italienischen Physiker Torricelli (1608-1647), dem bekannten Schüler Galileis, vorgelegt und von diesem auf mehrere Arten gelöst.

\section{Didaktische Informationen:}

b-11) Wir müssen darauf achten, dass der Extremwert wirklich existiert.

\section{Konstruktionen eines regelmässigen Vielecks mit Zirkel und Lineal.}

Vor der Untersuchung des Themas sind zwei unpassende Fragen in meinem Heft, die zu einer ungebundenden Rede gehörten und didaktisch interessant waren:

Was für eine Dichte haben die Primzahlen unter den natürlichen Zahlen?

Was können wir über die Zahl $\frac{\pi(n)}{n}$ sagen, wo die Zahl $\pi(n)$ die Anzahl der Primzahlen von 1 bis $n$ ist.

Die Einleitung des Themas ist das Problem von Delos (die Verdoppelung des Würfels: die Kante eines Würfels zu konstruieren, der doppelt so gross wie ein gegebener Würfel ist).

Der Satz von Gauss (1777-1855) ohne Beweis:

Ein reguläres Vieleck kann dann und nur dann mit Zirkel und Lineal konstruiert werden, wenn seine Seitenzahl die Form $2^{m} p_{1} p_{2} \ldots p_{k}$ hat, wo $p_{1}, p_{2}, \ldots, p_{k}$ untereinander verschiedene Primzahlen von der Form $2^{n}+1$ sind.

Es folgten zwei Sätze mit Beweis:

Wenn die Zahl $2^{n}+1\left(n \in \mathbb{N}^{+}\right)$eine Primzahl ist, dann hat die Zahl $n$ die Form $2^{m}\left(m \in \mathbb{N}^{+}\right)$.

Weil man das reguläre Dreieck und Fünfeck konstruieren kann, können wir auch das reguläre Fünfzehneck konstruieren. 


$$
\text { "kantor" — 2006/6/22 — 18:03 — page } 149 \text { — \#7 }
$$

Didaktische Informationen:

a-6) Bei der Untersuchung eines schweren Satzes ist es gut und genug, dass man die einfachen Teile des Satzes klarlegt.

b-12) Dieses Thema ist ein interessanter Abschnitt der Geschichte der Mathematik. Gauss sagte:

„Die Drei- und Fünfteilung des Kreises war schon zur Zeit Euklids bekannt; man muss sich wundern, dass jenen Entdeckungen in den seitdem verflossenen zwei Jahrtausenden nichts Neues hizugefügt wurde."

b-13) Der Satz der Konstruktion des Fünfzehnecks ist eine schöne Verkettung der Algebra und der Geometrie.

\section{Das Skalarprodukt der Vektoren.}

Die Definition und die einfachen Eigenschaften des Skalarprodukts der Vektoren. Eine Anwendung ist der Beweis des Satzes:

Die Höhen eines Dreiecks schneiden einander in einem gemeinsamen Punkt.

Didaktische Informationen:

b-14) Eine neue Methode für den Beweis eines bekannten Satzes.

\section{Eulers Gerade und der Feuerbach-Kreis.}

\section{Die Definition des Skalarproduktes der Vektoren.}

Betrachten wir die Vektoren $\mathbf{a}, \mathbf{b}, \mathbf{c}$, den Vektor e mit der Eigenschaft $|\mathbf{e}|=1$, und die Zahl $\lambda \in \mathbb{R}$. Wenn die folgenden Eigenschaften gelten, wo $\mathbf{a} \cdot \mathbf{b}$ ein Produkt der Vektoren $\mathbf{a}, \mathbf{b}$ bezeichnet und eine reelle Zahl bedeutet:

(1) $\mathbf{a} \cdot \mathbf{b}=\mathbf{b} \cdot \mathbf{a}$

(2) $\mathbf{a} \cdot(\mathbf{b}+\mathbf{c})=\mathbf{a} \cdot \mathbf{b}+\mathbf{a} \cdot \mathbf{c}$

(3) $(\lambda \mathbf{a}) \cdot \mathbf{b}=\lambda(\mathbf{a} \cdot \mathbf{b})$

(4) $\mathbf{e} \cdot \mathbf{e}=1$, dann gilt $\mathbf{a} \cdot \mathbf{b}=|\mathbf{a}| \cdot|\mathbf{b}| \cdot \cos (\mathbf{a}, \mathbf{b})$.

Didaktische Informationen:

b-15) Im Fachkreis habe ich gar nicht bemerkt, nur später ist es mir aufgefallen, dass ich damals die Geburt eines Satzes der Mathematik erlebt habe. Auch Tibor Szele hat sich mit diesem Thema (die Charakterisierung des Skalarproduktes der Vektoren) beschäftigt. 


$$
\text { "kantor" — 2006/6/22 — 18:03 — page } 150 \text { — \#8 }
$$

b-16) Dieses Thema steht mit der Definition der Potenzierung in Verbindung.

In seinem Lehrbuch „Tentamen“ definierte Farkas Bolyai (der Vater des Geometers János Bolyai) die Potenzen mit ganzen und rationalen Zahlen als Exponent auf Grund des Permanenzprinzips. Auch in diesem Fall ist die gewöhnliche Definition ein Satz.

b-17) In der höheren Mathematik sind ähnliche Charakterisierungssätze wichtig.

\section{Polyedersatz von Euler.}

Didaktische Informationen:

b-18) Die Methode des Beweises is beachtenswert, weil sie sich zu einem märchenhaften und anschaulichen Geschichtchen bindet. Die mit Dämmen voneinander getrennten Gebiete einer Insel sind zweckmässig mit Wasser zu überfluten. Der Beweis des Satzes folgt aus der Abzählung der Gebiete, der Ecken und der Dämme.

\section{Die pädagogische Prinzipien}

Zuletzt hebe ich noch zwei Ansichten hervor, die sich durch das ganze Material hindurchziehen und großartige Beispiele für die Vorstellung allgemeiner und spezieller pädagogischer Prinzipien sind.

I. Es ist von Fachkreisen der Mittelschule die Rede, weshalb viele Themen der höheren Mathematik und die dazugehörenden Mittel oft in Erscheinung treten. Leitprinzipien des Fachkreises waren

- der Aspekt des vorausgehenden Unterrichts: Vor der endgültigen Aufarbeitung des Materials die Vorbereitung mit geringerem Anspruch, der Leistung der Schüler entsprechend,

- der Aspekt des perspektivischen Unterrichts: Nach dem Unterricht auf niedrigerem Niveau muss man mit späterer Erweiterung und Vertiefung rechnen,

- die Konstruktion unterstützt den theoretischen Aspekt vor: Unterschiedliche Erkenntnisse und Untersuchungen während des Unterrichts.

II. Der Fachkreis hat einen erwähnungswerten und beispielhaften Inhalt. Begabte Schüler folgen der Lösung gut ausgewählter Themen, so wie 


$$
\text { "kantor" — 2006/6/22 — 18:03 — page } 151 \text { — \#9 }
$$

- grundlegend: Die Ausführungen und die Methoden gibt es sowohl in der angewandten als auch in der reinen Mathematik,

- elementar bzw. potenziell elementar: Gilt für die Form des Unterrichts,

- brauchbar: Die höhere Mathematik kennenzulernen, ist das Ziel des Fachkreises.

SÁNDOR KÁNTOR

INSTITUTE OF MATHEMATICS

UNIVERSITY OF DEBRECEN

H-4010 DEBRECEN

P. O. BOX 12

HUNGARY

E-mail: kantor@math.klte.hu

(Received November, 2005) 